\title{
étude en laboratoire des états limites d'une argile molle organique
}

\author{
par \\ J.-P. Magnan, \\ S. Shahanguian, \\ H. Josseaume
}

\section{Introduction}

Le développement des méthodes de calcul numérique, et notamment de la méthode des éléments finis, a conduit au cours des dernières années la mécanique des sols à une situation où les méthodes de calcul sont très puissantes sans que l'on ait une connaissance équivalente des lois de comportement des sols et oủ le perfectionnement des méthodes de calcul ne présente de ce fait dans l'immédiat qu'un intérêt limité. La nécessité d'une priorité aux études rhéologiques a été ressentie également au Laboratoire Central des Ponts et Chaussées oủ des recherches expérimentales en laboratoire ont été conduites parallèlement au développement des méthodes de calcul (Dang et Magnan, 1977; Magnan et Belkeziz, 1982; Magnan et Mouratidis, 1981) et à la réalisation d'études en vraie grandeur sur le site expérimental de remblais sur argile molle de Cubzac-les-Ponts (Blondeau et al, 1977; Vogien, 1975; Magnan et al, 1978).

L'étude expérimentale dont il est rendu compte dans cet article a été lancée en 1977, après le $9^{\circ}$ Congrès International de Mécanique des Sols et des Travaux de Fondation de Tokyo, au cours duquel Tavenas et Leroueil (1977) ont présenté les premiers résultats de leurs travaux sur la rhéologie des argiles de la vallée de Saint Laurent, analysée en termes d'état-limite et d'état-critique, c'est-à-dire dans l'esprit du modèle élaboré au cours des années soixante par le groupe de Mécanique des Sols de l'Université de Cambridge. sous la conduite du professeur Roscoe.

Les modèles de Cambridge pour les argiles (Cam-Clay, Cam-Clay modifié, Cam-Clay modifié révisé) ont été développés entre 1963 et 1967 (Roscoe et al, 1963; Roscoe et Poorooshasb, 1963; Burland, 1965, 1967) par combinaison de concepts élaborés antérieurement par Rendulic (1936) et Hvorslev (1936) pour la surface de chargement, par Roscoe et al (1958) pour l'état critique et par Drücker $(1951,1964)$ pour les conditions de stabilité, quî s'appliquent à l'écoulement du sol sur la surface de chargement. Ils ont fait l'objet de vérifications expérimentales sur du kaolin reconstitué (Roscoe et Hambly, 1969; Balasubramaniam, 1969; Pearce, 1971) et d'une adaptation pour traitement par la méthode des éléments finis par Zienkiewicz et Naylor (1971) et Simpson (1973).

Les recherches effectuées à I'Université Laval de Québec par F. Tavenas et S. Leroueil ont confirmé l'intérêt des notions d'états limite et critique pour la description du comportement en laboratoire des argiles sensibles du Québec mais conduit à des courbes d'état limite (ou surfaces de chargement) plus ou moins centrées sur l'axe d'anisotropie (" $\mathrm{K}_{0}$ ") du sol et non pas sur l'axe des contraintes isotropes, comme c'était le cas pour les modèles de Cambridge.

Le Laboratoire Central des Ponts et Chaussées était au courant de l'avancement de ces travaux dans le cadre d'un projet de coopération franco-québécoise qui l'unissait à I'Université Laval et il fut décidé à cette époque de tester sur une argile molle française les nouveaux concepts élaborés à l'Université Laval (modèle YLIGHT). Le choix s'est porté sur l'argile molle organique du site expérimental de Cubzac-les-Ponts.

L'étude a porté sur la détermination de la surface d'état limite de cette argile molle organique, que l'on voulait utiliser ultérieurement pour des calculs en éléments finis. Elle a été réalisée sur des éprouvettes de sol prélevées à trois endroits du site.

La suite de cet article présente successivement :

- les objectifs de l'étude et la stratégie adoptée;

- la procédure et le matériel utilisés pour les essais;

- le sol testé (argile molle organique de Cubzac-lesPonts);

- les résultats obtenus (chemins de contraintes et courbes d'état-limite);

- les prolongements souhaitables de la recherche. 


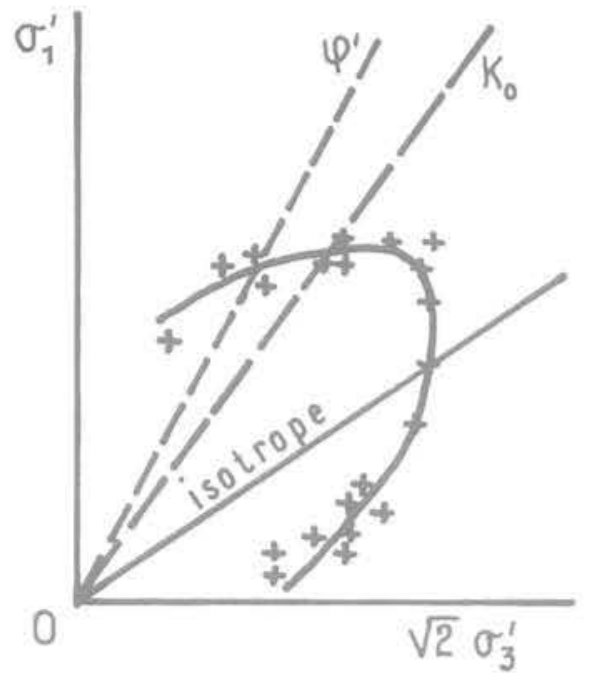

a- Heron Road - Bronson Ave

(Wong et Mitchell, 1975)

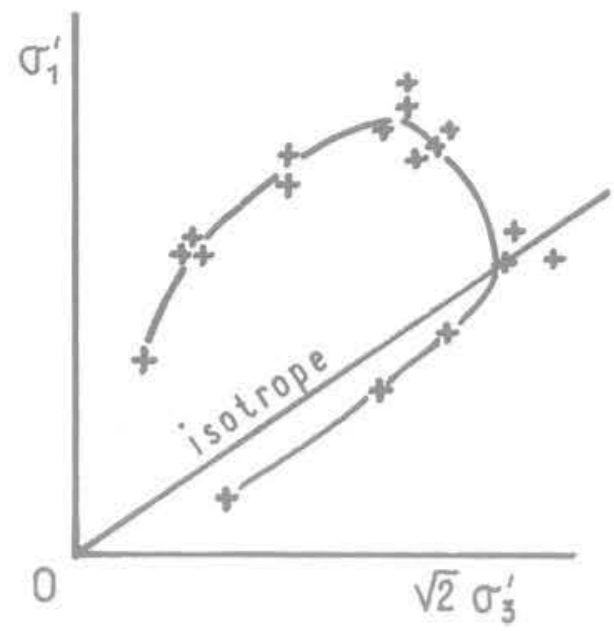

c-Drammen (Berre, 1972)

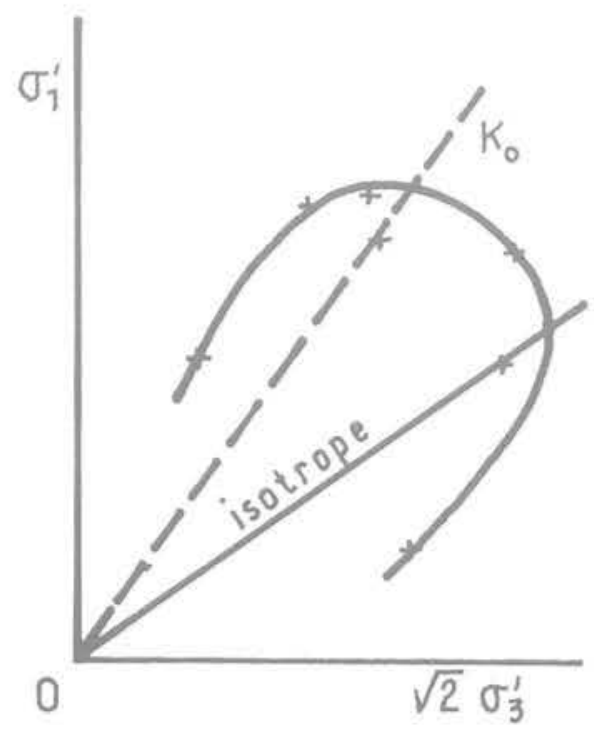

e-Mastemyr (Graham,1969)

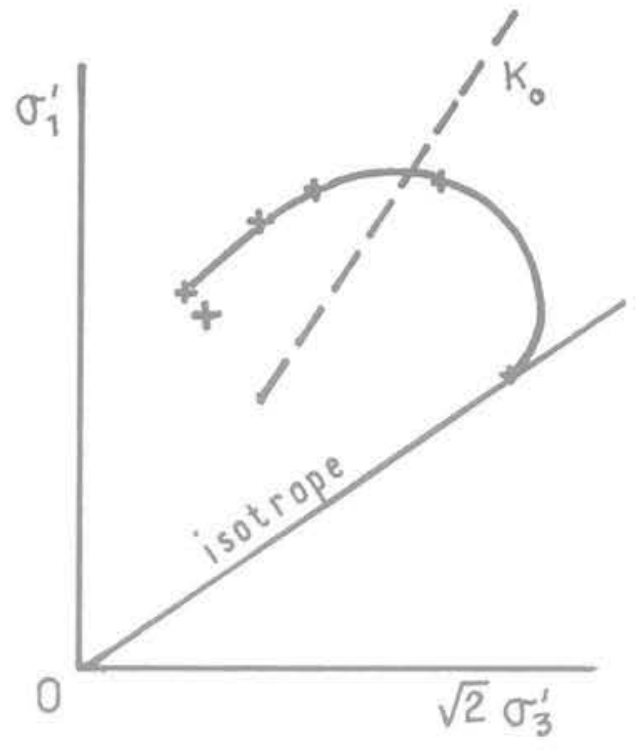

b-Lyndhurst (Graham,1974)

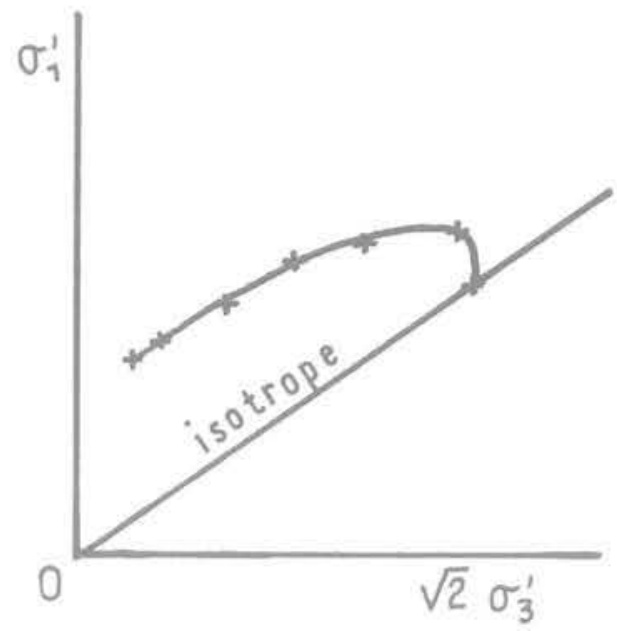

d-Ottawa (McRostie et al, 1972)

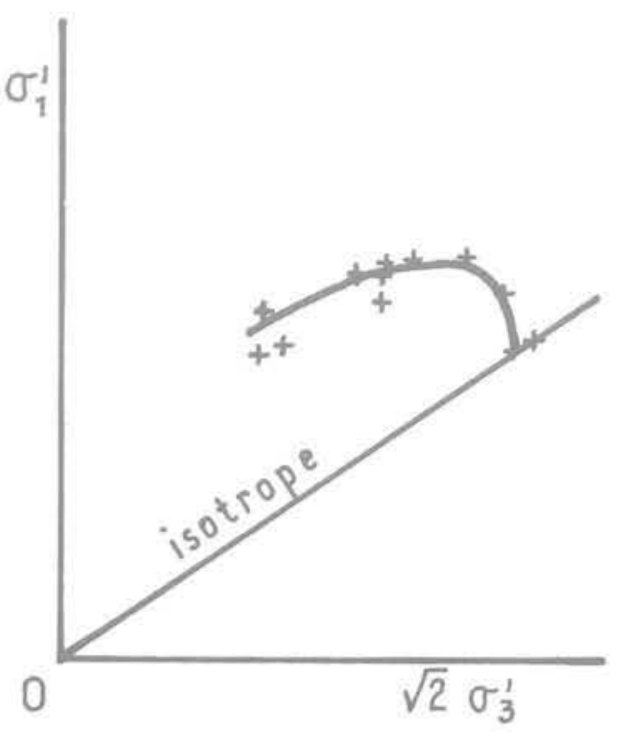

f-Rockcliffe (Mitchell, 1970)

Fig. 1 Allure des courbes d'état limite de différentes argiles naturelles (d'après Leroueil, 1977) 


\section{Objectifs de l'étude et stratégie adoptée}

L'existence de courbes d'état limite dans les argiles molles est attestée par les exemples rassemblés par Leroueil (1977) et présentés sur les figures 1 et 2.

Chacune de ces courbes définit, pour un état initial donné, l'ensemble des états de contraintes que le sol peut subir sans qu'il se produise de déformations irréversibles importantes. L'ensemble des courbes d'état limite correspondant aux états initiaux possibles, caractérisés généralement par l'indice des vides du sol, constitue la surface d'état limite.

Cette définition appelle plusieurs remarques:

- d'une part, tous les points d'une courbe d'état limite ne correspondent pas à des irrèversibilités de même nature (fig. 3 ). Au-dessus de la droite de pente $\mathrm{M}$ qui correspond dans le repère $(\mathrm{s}, \mathrm{t})$ à la condition classique de Mohr-Coulomb

$\tau_{\max }=c^{\prime}+\sigma^{\prime} \operatorname{tg} \varphi^{\prime}$ ou $\varnothing^{\prime}$ par $\varnothing^{\prime} !$

on observe une rupture du sol par cisaillement, tandis quau dessous de cette droite il apparaît des déformations volumiques irréversibles, comme iorsque l'on dépasse la pression de préconsolidation dans un essai œdométrique:

- d'autre part, on peut utiliser différentes représentations pour les courbes d'état limite : repères $\left[\mathrm{s}=0,5\left(\sigma_{1}^{\prime}+\sigma_{3}^{\prime}\right), \mathrm{t}=0,5\left(\sigma_{1}^{\prime}-\sigma_{3}^{\prime}\right)\right]$,

$\left[\sigma_{1}^{\prime}, \sqrt{2} \sigma_{3}^{\prime}\right]$ ou

$\left[p=0,333\left(\sigma_{1}^{\prime}+2 \sigma_{3}^{\prime}\right), q=\sigma_{1}^{\prime}-\sigma_{3}^{\prime}\right]$,

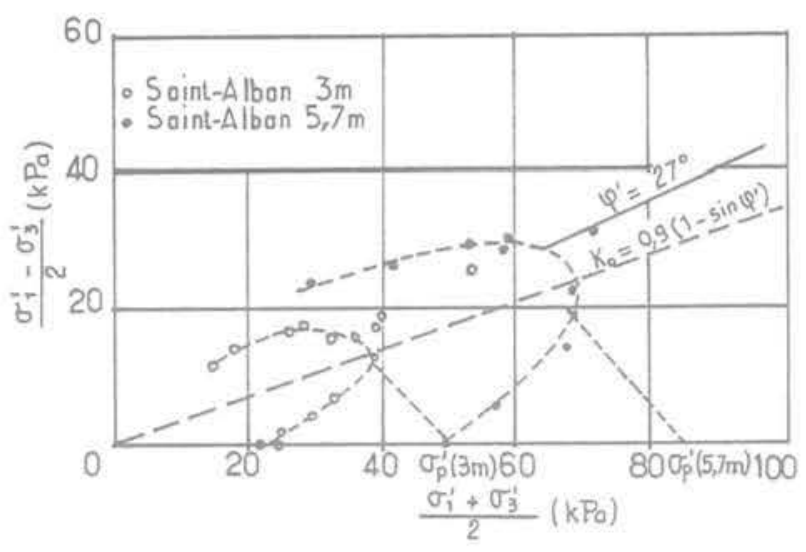

Fig. 2 Courbes d'état limite de l'argile de Saint-Alban (d'après Leroueil, 1977)

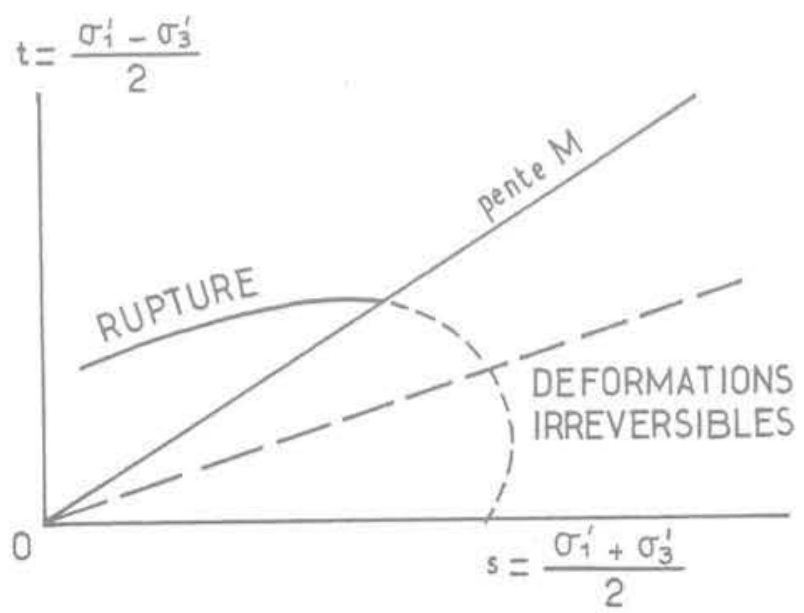

Fig. 3 Nature des irréversibilités sur la courbe d'état limite par exemple.

La forme des courbes d'état limite s'en trouve modifiée (fig. 4), mais de façon peu importante. compte tenu du caractère linéaire des relations entre ces différents systèmes de coordonnées:

- enfin, les seuls paramètres utilisés sont $\sigma_{1}^{\prime}, \sigma_{3}^{\prime}$ et e, ce qui fait que ce mode de représentation est incapable de traduire l'influence éventuelle de la contrainte effective principale intermédiaire $\sigma_{2}^{\prime}$ lorsqu'elle diffère de $\sigma_{3}^{\prime}$, des rotations des axes de contraintes, de la distorsion du sol, etc., sauf à introduire un paramétrage des surfaces d'état limite obtenues.

L'étude effectuée Laboratoire Central des Ponts et Chaussées a été réalisée, comme celles qui l'ont précédée, sur du matériel triaxial et cedométrique classique, c'est-à-dire à symétrie de révolution, de telle sorte qu'une représentation dans un système $(s, t)$ était tout à fait suffisante.

Pour déterminer les points de la courbe d'état limite correspondant à un état initial donné du sol, on a réalisé des essais de différents types, dont on a analysé le déroulement en termes de contraintes effectives, le point de contraintes $(\mathrm{s}, \mathrm{t})$ correspondant au phénomène attendu (rupture ou accélération des déformations) étant supposé sur la courbe d'état limite. La figure 5 présente les chemins de contraintes correspondant aux différents types d'essais réalisés

- essais triaxiaux $\mathrm{K}_{0}$ :

- essais triaxiaux consolidés de façon isotrope et non drainés (CIU) avec mesure de la pression interstitielle:

- essais triaxiaux consolidés de façon isotrope et drainés (CID);

- essais triaxiaux de striction, avec consolidation anisotrope:

- essais de consolidation anisotrope (à $\sigma_{3}^{\prime} / \sigma_{1}^{\prime}=\mathrm{K}=$ constante).
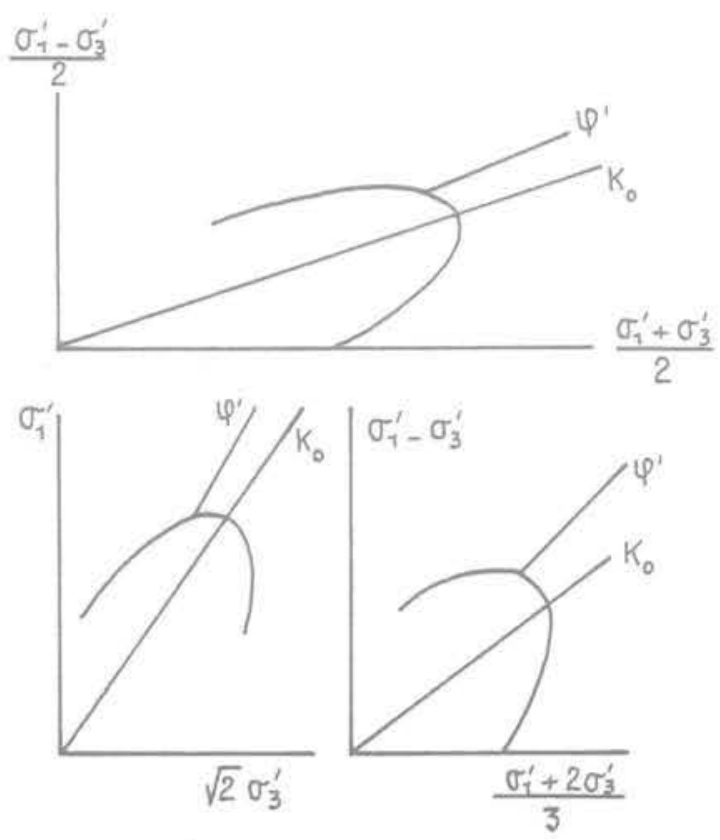

Fig. 4 Influence du mode de représentation sur la forme des courbes d'état limite 


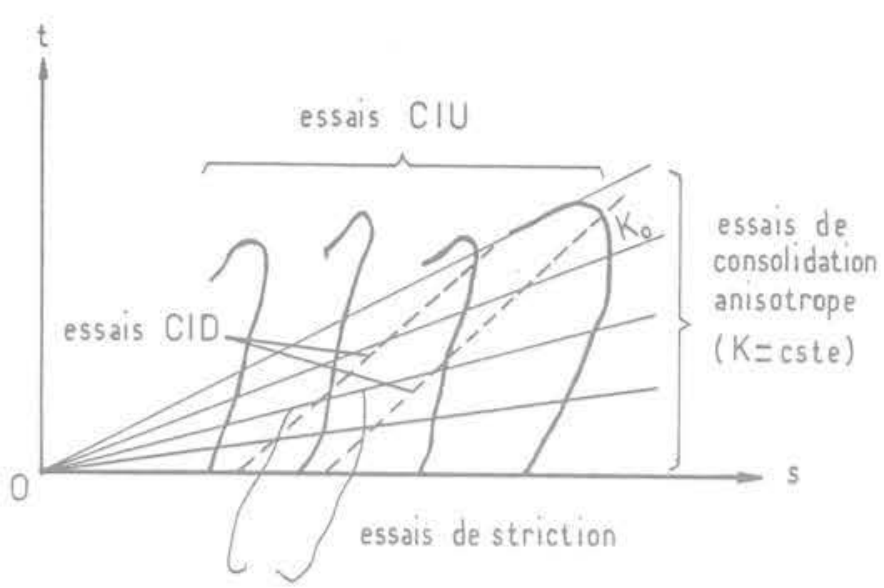

Fig. 5 Chemins de contraintes suivis pendant les essais

Des essais œdométriques ont également été réalisés mais, comme pour ce type d'essais on ne peut jamais être totalement sûr du respect de la condition $\mathrm{K}_{0}$, on ne connait pas de façon certaine la valeur du rapport $\sigma_{3}^{\prime} / \sigma_{1}^{\prime}$. On a reporté pour cette raison sur les diagrammes le lieu des points correspondant aux contraintes de préconsolidation, soit $\sigma_{1}^{\prime}=\sigma_{\mathrm{p}}^{\prime}$, pour toutes les valeurs possibles du rapport $\sigma_{3}^{\prime} / \sigma_{1}^{\prime}$.

Pour les essais de cisaillement, on a retenu les pics des chemins de contraintes tandis que, pour les essais de consolidation, le point limite adopté correspond au coude de la courbe $\propto$ variation de volume $=f$ (contrainte effective moyenne) * représentée en coordonnées línéaires.

Pour chaque point d'étude du sol de Cubzac-les-Ponts, on a réalisé un nombre d'essais de chaque type suffisant pour obtenir la forme de la courbe d'état limite.

\section{Procédures et matériels d'essai}

Les essais triaxiaux ont été réalisés sur du matériel (cellule et presse) classique de marque WykehamFarrance. On a travaillé sur des éprouvettes de $50,6 \mathrm{~mm}$ de diamètre et d'élancement 2 (soit une hauteur de $101,2 \mathrm{~mm}$ ). Pour la mesure des déformations latérales des éprouvettes, on a utilisé les bagues à vernier conçues par B. Félix (1981) ainsi que les burettes classiques pour la mesure des volumes drainés. Les mebranes entourant les éprouvettes étaient des membranes en latex d'épaisseur nominale 2/10 mm fabriquées par Wykeham-Farrance. La cellule était remplie d'huile de silicones Rhodorsil $47 \mathrm{~V} 300$ fabriquée par I Société Rhône-Poulenc.

La mise en charge des essais de striction et des essais $K_{0}$ a été effectuée à l'aide des dispositifs spéciaux mis au point lors de recherches antérieures (Josseaume et Meimon, 1976).

Le chargement des éprouvettes soumises aux essais de consolidation anisotrope a été réalisé manuellement, ce qui peut expliquer certaines irrégularités locales des chemins de contraintes effectives. Par contre, toutes les mesures de déplacements, de contraintes totales et de pressions interstitielles ont été enregistrées en continu, le dépouillement des résultats s'effectuant sur ordinateur.

Les essais triaxiaux ont été réalisés avec une contre-pression de 34 à $48 \mathrm{kPa}$ appliquée préalablement au début du chargement et correspondant approximativement aux pressions interstitielles en place.

Pour les essais œdométriques, on a utilisé indifféremment des œdomètres à poids de marque Wykeham Farrance ou des œdomètres pneumatiques de type LPC.

\section{Le sol testé (argile molle organique de Cubzac-les-Ponts)}

Les éprouvettes utilisées pour les essais proviennent de carottes prélevées en deux endroits du site expérimental de Cubzac-les-Ponts

- à l'emplacement du rembiai $\mathrm{C}$ on a prélevé des carottes de diamètre $76 \mathrm{~mm}$ à l'aide du carottier à piston stationnaire LPC. Les essais ont été réalisés autour de deux niveaux moyens : $-4,5 \mathrm{~m}$ (carottes de 4 à $5 \mathrm{~m}$ ) et $-5,5 \mathrm{~m}$ (carottes de 5 à $6 \mathrm{~m}$ ). Les sondages correspondants portent les numéros $\mathrm{A} 35$, A36, $A 37$ et $A 38$ :

- à l'emplacement du remblai D on a prélevé des carottes de $200 \mathrm{~mm}$ de diamètre à l'aide du carottier développé par l'Université Laval. Ces carottes sont découpées en blocs de $13 \mathrm{~cm}$ de hauteur dès leur extraction et conservées à l'aide d'une couche de cire et de paraffine appliquée sur le site même du prélèvement. Les essais ont été réalisés sur des blocs prélevés aux environs de $5.5 \mathrm{~m}$ de profondeur. Les sondages portent les numéros A40 et A41.

Les caractéristiques moyennes de l'argile molle testée, sont, pour les trois emplacements testés :

- teneur en eau w comprise entre 60 et 80 , toujours très proche de la limite de liquidité $w_{L}$ :

- indice de plasticité $I_{\mathrm{p}}$ voisin de 40 ;

- indice des vides initial $e_{0}$ de l'ordre de 2,5;

- sol légèrement surconsolidé (d'une dizaine de kilopascals).

\section{Résultats}

Les figures 6,7 et 8 présentent, pour chacune des trois zones étudiées (remblai C/ $-4,5 \mathrm{~m}$; remblai $\mathrm{C} /-5,5 \mathrm{~m}$; remblai $\mathrm{D} /-5,5 \mathrm{~m}$ ), d'une part les chemins de contraintes effectives suivis lors des essais de cisaillement à l'appareil triaxial (essais CIU et CID), ainsi que l'enveloppe de rupture obtenue en joignant les pics de ces différentes courbes, et d'autre part l'ensemble des points de la courbe d'état limite (pics précédents plus les points correspondant à l'accélération des tassements dans les essais de consolidation anisotropes). La droite $\sigma_{1}^{\prime}=\sigma_{p}^{\prime}$ de ce second diagramme correspond au résultat moyen des essais œdométriques.

Les résultats sont très encourageants, même si certains points ne sont pas connus avec une précision très élevée (la mesure des variations de volume donne souvent des résultats différents selon le système utilisé, bagues ou burettes, tandis que les corrections de membranes et autres peuvent influer sur les résultats des essais de cisaillement).

Le seul point "anormal " des résultats est la position de la droite $\sigma_{1}^{\prime}=\sigma_{p}^{\prime}$ par rapport à la courbe d'état limite dans deux des cas (remblai C à $-4,5$ et $-5,5 \mathrm{~m}$ ) : cette droite coupe la courbe d'état limite alors qu'elle devrait seulement la toucher sur la droite de consolidation anisotrope sous $\sigma_{3}^{\prime} / \sigma_{1}^{\prime}=K_{0}$ si l'essai œdométrique était 

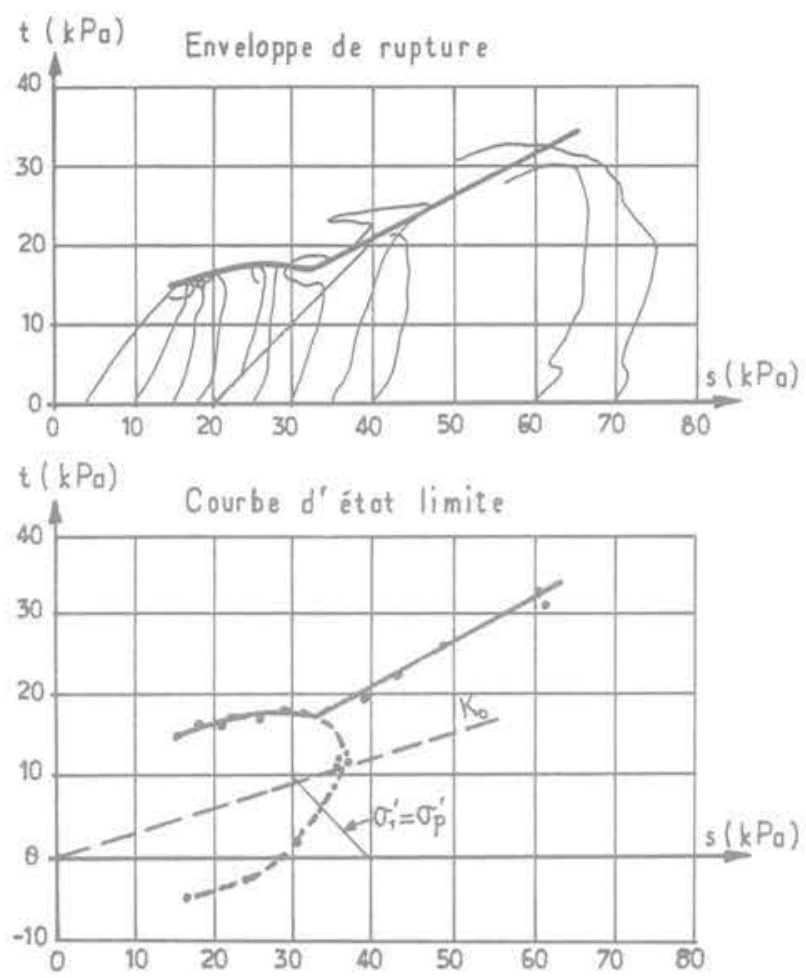

Fig. 6 Courbe d'état limite et enveloppe de rupture (remblai $C ;-4,5 \mathrm{~m}$ )
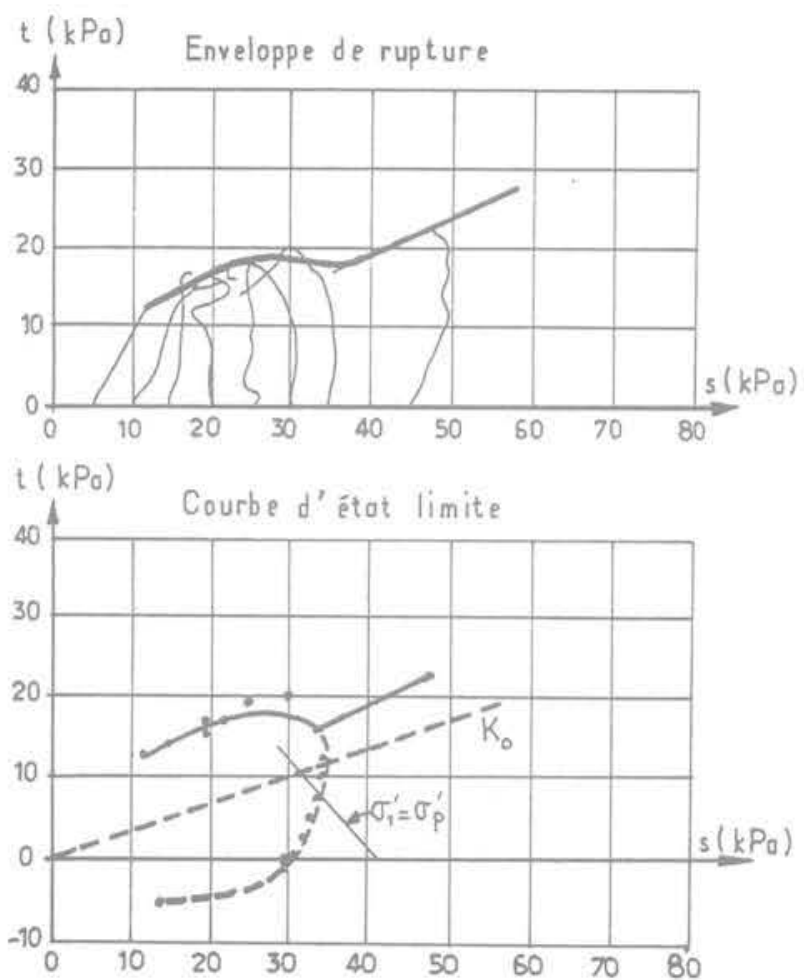

Fig. 7 Courbe d'état limite et enveloppe de rupture (remblai $C ;-5,5 \mathrm{~m}$ )

vraiment un essai $K_{0}$ c'est-à-dire à déformation horizontale nulle. Cette anomalie mériterait certainement une étude approfondie.
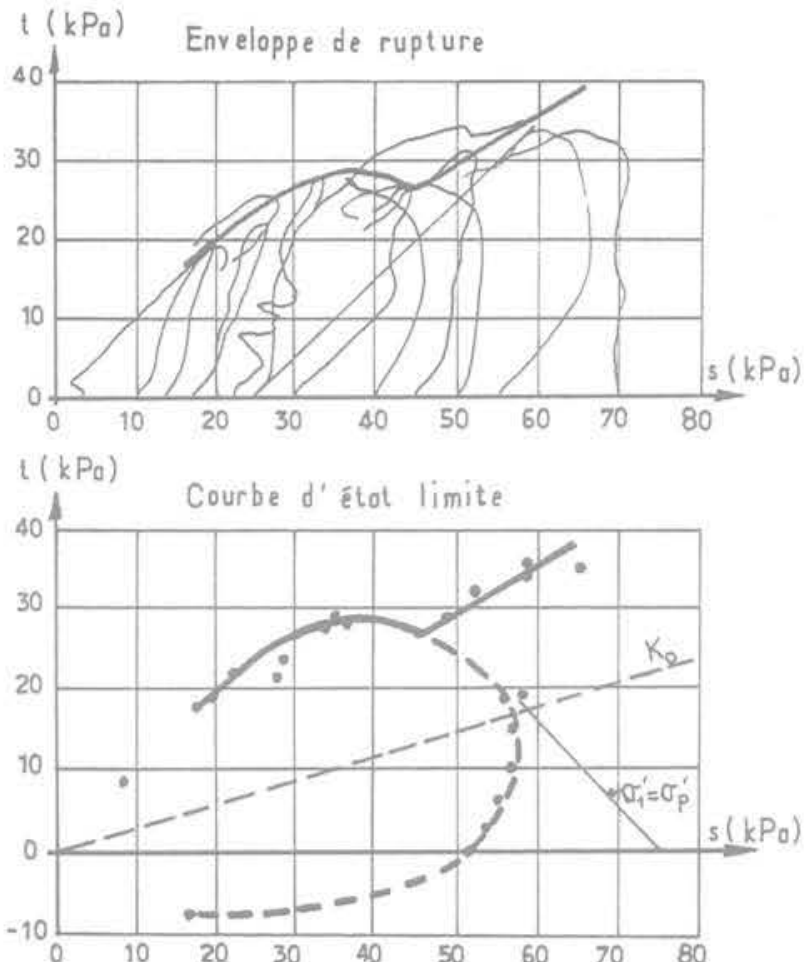

Fig. 8 Courbe d'état limite et enveloppe de rupture (remblai $D ;-5,5 \mathrm{~m}$ )

Les résultats obtenus par Leroueil (1977) sur l'argile de Saint-Alban confirmaient une des hypothèses principales du modèle testé $i i_{1}$ à savoir le caractère homothétique des courbes obtenues sur des blocs d'argile d'état initial différents. La normalisation des courbes d'état limite par rapport à la contrainte de préconsolidation $\sigma_{0}^{\prime}$ doit conduire à une courbe d'état limite unique si cette hypothèse est vraie. La figure 9 présente l'ensemble des résultats de l'étude dans un système de coordonnées réduites ou "normalisées" $\left(s^{*}=s / \sigma_{p}^{\prime} ; t^{*}=t / \sigma_{p}^{\prime}\right)$. Il est clair que les courbes peuvent être considérées comme homothétiques puisque l'on peut définir sans peine sinon une courbe unique, du moins une bande d'ètat limite de largeur faible et tolérable compte tenu des incertitudes inhérentes à ce type d'étude en laboratoire (fig. 10). II convient de noter, toutefois, que la contrainte de préconsolidation $\sigma_{p}^{\prime}$ utilisée pour la normalisation des résultats expérimentaux n'est pas celle des essais œdométriques mais la valeur correspondant aux points de la courbe d'état limite situés sur la droite d'anisotropie $\mathrm{K}_{0}$.

Un dernier aspect des résultats mérite un commentaire : les points alignés sur la droite appelée $\phi^{\prime}$ sur la figure 10 correspondent aux essais de cisaillement $\mathrm{CIU}$ consolidès sous des pressions isotropes supérieures à la pression de préconsolidation du sol. En toute rigueur, ces points ne devraient pas figurer dans les diagrammes de résultats puisqu'ils ne proviennent pas d'éprouvettes de même état initial que les autres. Nous les avons laissés parce que toutes les publications antérieures comportent des points analogues. Les chemins de contraintes correspondants ne sont d'ailleurs pas dépourvus d'intérêt: d'après les principes généraux du modèle de Tavenas et Leroueil (1979). ils donnent une idée de la forme de la surface d'état limite pour les pressions de préconsolidation supérieures à la pression de préconsolidation du sol naturel. 


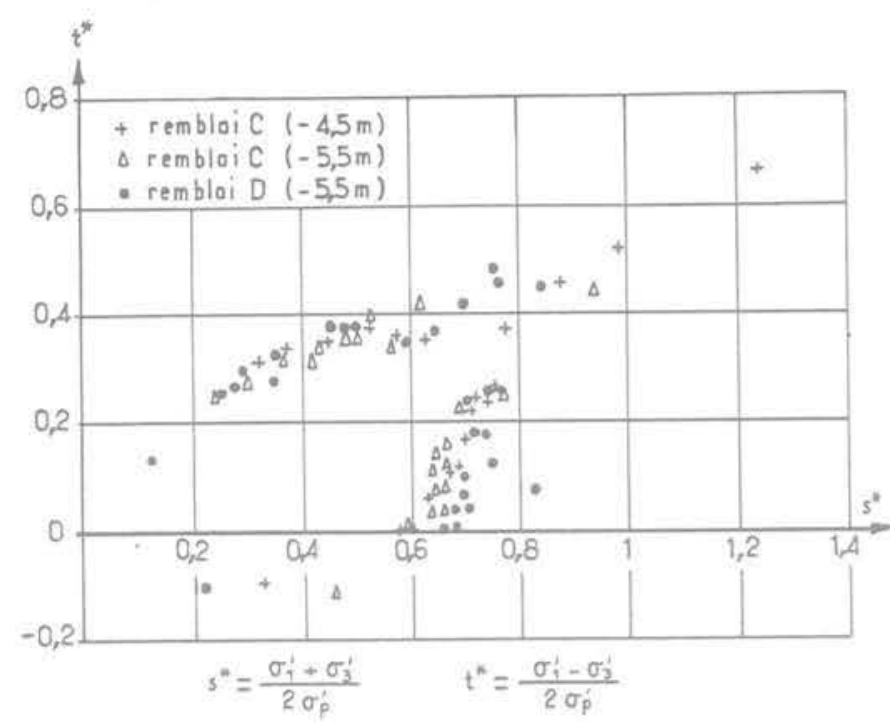

Fig. 9 Résultats des essais en coordonnées "normalisées»

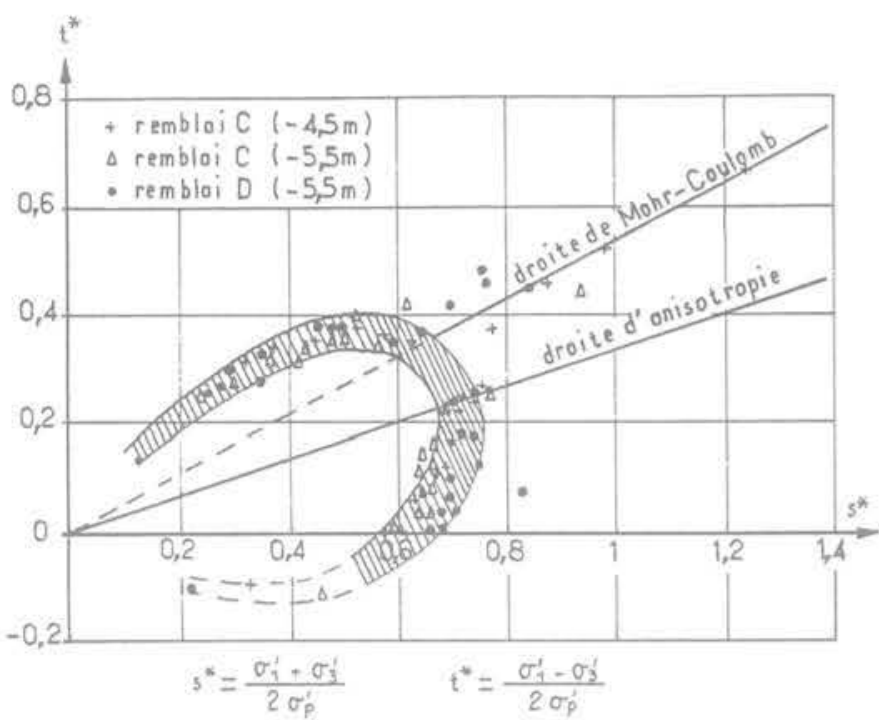

Fig. 10 Courbe d'état limite de l'argile organique de Cubzac-les-Ponts

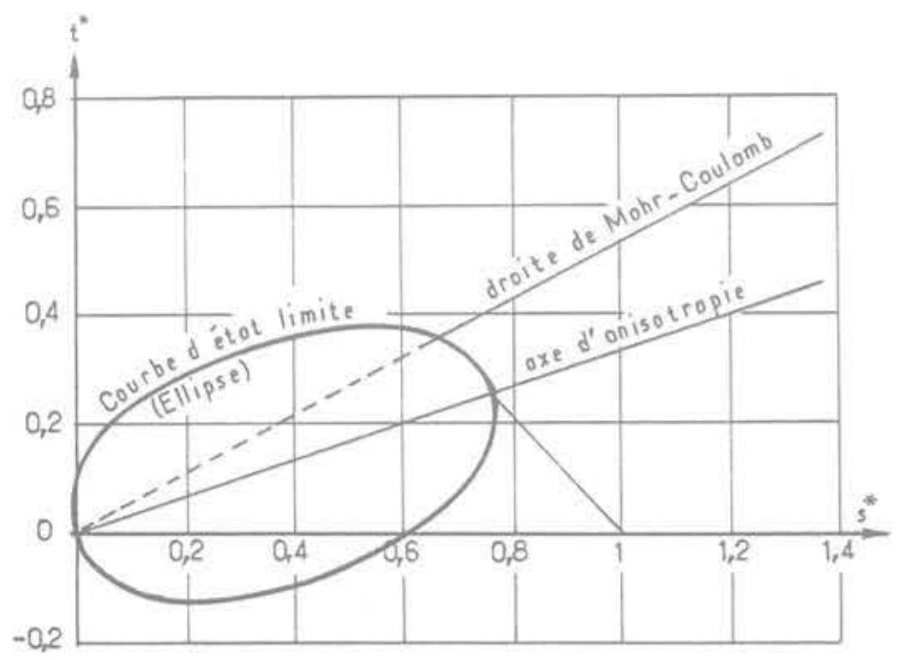

Fig. 11 Représentation schématique des courbes d'état limite
La figure 11 présente la schématisation adoptée à la suite de cette étude pour les courbes d'état limite du sol de Cubzac, définies à l'aide d'une courbe unique dans le repère normalisè $\left(\mathrm{s}^{*}, \mathrm{t}^{*}\right)$.

Les résultats détaillés des essais sont disponibles dans la thèse de Shahanguian (1980).

\section{Perspectives et conclusion}

La détermination de la géométrie des courbes d'état limite est un premier pas important pour le développement de modèles élastoplastiques avec écrouissage adaptés au comportement des argiles naturelles. Mais d'autres informations sont nécessaires si l'on veut transformer ces courbes d'état limite en modèle de calcul. II faut connaître notamment

- le comportement du sol à l'intérieur de la surface d'état limite:

- Ia loi d'écoulement, associée ou non associée, à utiliser sur la surface d'état-limite.

II faut également préciser le mode de variation éventuel de la forme de la surface limite lors de l'écrouissage. des études réalisées à l'Université Laval (Brucy, 1977; Leroueil et al, 1979) semblent indiquer que, pour les argiles sensibles de la vallée du Saint Laurent, il existe une surface d'état limite initiale ayant une forme d'allure elliptique mais que cette surface se déforme dès que l'on atteint la surface lors du chargement : la partie de la courbe située au-dessus de la droite de rupture $\phi^{\prime}$ tend à se rabattre vers cette droite tandis que la partie de la courbe située au-dessous de la droite de rupture prend progressivement une forme différente qui dépend du type de sollicitation imposée au sol (par exemple, si l'on réalise une compression isotrope du sol, la courbe d'état limite tend à devenir symétrique par rapport à l'axe Os).

Enfin, l'influence de la rotation des axes des contraintes principales par rapport aux axes "géométriques " ou "physiques" du matériau doit être étudiée.

Toutes ces ètudes, dont certaines sont en cours, sont indispensables au progrès de la rhéologie des sols.

\section{Références bibliographiques}

A.S. Balasubramaniam, (1969) Some factors influencing the stress-strain behaviour of clay. Ph. D. Thesis, Cambridge University.

T. Berre, (1972) Sammenheng mellom tid, deformasjoner og spenninger for normalkonsoliderte marine leirer. Proc. Nordic Conf. on Soil Mechanics, Trondheim.

F. Blondeau, C. Mieussens, D. Queyroi, J.-P. Levillain, M. Peignaud (1977) Instrumentation du remblai expérimental A de Cubzac-les-Ponts. International Symposium on Soft Clay, Bangkok, 419-435, et Bull, Liaison Labo. Ponts et Chaussées, $n^{\circ}$ spécial VI-F et VI-E.

F. Brucy, (1977) Effets du passage en l'état normalement consolidé sur l'état limite d'une argile naturelle. Thèse de maîtrise. Université Laval, Québec, $167 \mathrm{p}$.

J.P. Burland, (1965) The yielding and dilation of clay. Correspondence. Geotechnique, vol. 15, 211-214.

J.P. Burland, (1967) Deformation in soft clay. Ph. D. Thesis, Cambridge University. 
M. T. Dang, J.P. Magnan, (1977) Application des modèles élastoplastiques de I'Université de Cambridge au calcul du comportement d'un remblai expérimental sur sols mous. Laboratoire Central des Ponts et Chaussées, Paris, Rapport de recherche LPC $n^{\circ} 74$. 106 pages.

D.C. Drücker (1951) A more fundamental approach to plastic stress-strain relations. Proc. 1 st US National Congress on Applied Mechanics ASCE, Journal of Soil Mech. and Foundn division.

D.C. Drücker, (1964) On the postulate of stability of material in the mechanics of continua. Journal de Mécanique. Vol. 3, 235-249.

B. Fèlix, (1981) Mesure de la déformation radiale des éprouvettes de sol par un système de bagues. Revue Française de Géotechnique, $n^{\circ} 15$.

J. Graham, (1969) Laboratory results from Mastemyr quick clay after reconsolidation to the in situ stresses. Institut Géotechnique Norvégien, Oslo, Rapport interne, 372-375

J. Graham, (1974) Laboratory testing of sensitive clay from Lyndhurst, Ontario. Civil Engng Research Report 74-2, Royal Military College, Kingston, Ontario, Canada.

M.J. Hvorslev, (1936) Conditions of failure for remoulded cohesive soils. Discussion. C.-R. $1^{\text {er }}$ Congrès International de Mécanique des Sols. Cambridge, Mass., vol. 3, 51-53.

H. Josseaume, Y. Meimon, (1976) Détermination de la loi de comportement des argiles molles en laboratoire. Bull. Liaison Labo. Ponts et Chaussées, n Spécial III, décembre $1976,117-127$.

S. Leroueil, (1977) Quelques considérations sur le comportement des argiles sensibles. Thèse de Doctorat-ès-Sciences, Universitè Laval, Québec.

S. Leroueil, F. Tavenas, F. Brucy, P. La Rochelle, M. Roy, (1979) Behaviour of destructured natural clays. Proc. ASCE, Journal of Geotechnical Engineering Division, vol. 105, n GT6, 759-778.

J. P. Magnan, A. Belkeziz, (1982) Consolidation d'un sol élastoplastique. Revue Française de Géotechnique. A paraitre.

J. P. Magnan, C. Mieussens, D. Queyroi, (1978) Comportement du remblai expérimental B à Cubzac-lesPonts. Revue Française de Géotechnique, $n^{\circ} 5$, Nov. $1978,23-36$

J. P. Magnan, A. Mouratidis, (1981) Déformations et contraintes dans un massif de sol décrit par le modèle Cam-Clay modifié. Colloque de Rhéologie. École Nationale des Travaux Publics de l'État, Lyon.

G.C. Mc Rostie, K.N, Bur, R.J. Mitchell, (1972) The performance of tied-back sheet piling in clay. Canadian Geotechnical Journal, vol. 9, $n^{\circ} 2,206-218$.

R.J. Mitchell, (1970) On the yielding and mechanical strength of Leda clays. Revue Canadienne de Géotechnique, vol. $7, n^{\circ} 3,297-312$.
J.A. Pearce, (1970) The behaviour of soft clay in a new true triaxial, Ph. D. Thesis, Cambridge University.

L. Rendulic, (1936) Relation between void ratio and effective principal stresses for a remoulded silty clay. Discussion C.-R. $1^{\text {er }}$ Congrès International de Mécanique des Sols, Cambridge, Mass., vol. 3, 186-191.

K.H. Roscoe, A.N. Schofield, C.P. Wroth, (1958) On the yielding of soils. Géotechnique, vol. 8, n 1, 22-53.

K.H. Roscoe, H.B. Poorooshasb, (1963) A theoretical and experimental study of strains in triaxial compression test on normally consolidated clays. Géotechnique, vol. $13, n^{\circ} 1,12-38$.

K.H. Roscoe, A.N. Schofield, A. Thurairajah, (1963) Yielding of clays in states wetter than critical. Géotechnique, vol. 13, $n^{\circ} 2,211-240$.

K.H. Roscoe, J.B. Burland, (1967) On the generalized stress-train behaviour of "wet" clay. Symposium on engineering plasticity. Cambridge Univ. Press, 53̣5-609.

K. H. Roscoe, E.C. Hambly, (1969) Observations and predictions of stresses and strains during plane strain of "wet clay". C.-R. $7^{\circ}$ Congrès International de Mécanique des Sols et des Travaux de Fondation. Mexico, vol. 1, 17-181.

D.A. Sangrey, (1972) Naturally cemented sensitive soils. Géotechnique, vol. $22, n^{\circ} 1,139-152$.

S. Shahanguian, (1980) Loi de comportement des argiles molles: détermination expérimentale des courbes d'état limite de l'argile organique de Cubzacles-Ponts. Thèse de docteur-ingénieur. Université Pierre et Marie Curie (Paris VI), 180 pages : Laboratoire Central des Ponts et Chaussées, Paris, Rapport de Recherche LPC n०106, 1981.

B. Simpson, (1973) Finite elements applied to problems of plane strain deformation in soils. Ph. D. Thesis. Cambridge University.

F. Tavenas, S. Leroueil, (1977) Effects of stresses and time on yielding of clays. C.-R. $9^{e}$ ongrès Internationa de Mécanique des Sols et des Travaux de Fondation. Tokyo, vol. 1, 319-326.

F. Tavenas, S. Leroueil, (1979) Les concepts d'état limite et d'état critique et leurs applications pratiques à l'étude des argiles. Revue française de Géotechnique, $n^{\circ} 6$, février 1979, 27-49.

M. Vogien, (1975) Etude du comportement avant la rupture d'un remblai expérimental construit sur sol mou à Cubzac-les-Ponts. Thèse de docteur-ingénieur. Université Pierre et Marie Curie, Paris.

P.K.K. Wong, R.J. Mitchell, (1975) Yielding and plastic flow of sensitive cemented clay. Géotechnique, vol. 25 , $n^{\circ} 4,763-782$

O.C. Zienkiewicz, O.J. Naylor, (1971) Discussion of the adaptation of critical state soil mechanics theory for use in finite elements. Proc. Roscoe Memorial Symposium. Cambridge University, 537-547. 
